\title{
Approximating Arbitrary Fuzzy Subsets by Extensional Ones
}

\author{
G. Mattioli \\ Secció Matemàtiques i Informàtica \\ ETSAV - UPC \\ Pere Serra 1-15, 08190 \\ Sant Cugat del Vallès, Spain \\ gabriel.mattioli@upc.edu \\ J. Recasens \\ Secció Matemàtiques i Informàtica \\ ETSAV - UPC \\ Pere Serra 1-15, 08190 \\ Sant Cugat del Vallès, Spain \\ j.recasens@upc.edu
}

\begin{abstract}
An interesting problem within the theory of indistinguishability operators is how to approximate an arbitrary fuzzy subset by a similar extensional one. In this paper the authors aim at solving this question and provide three methods to find extensional approximations of fuzzy subsets $\mu$. These methods are exhaustively explained for different Archimedean t-norms and an example is provided to illustrate them.

Keywords: Indistinguishability Operator, Extensional Fuzzy Subset, Upper Approximation, Lower Approximation, Quasi-arithmetic mean, Power with respect to a $t$-norm
\end{abstract}




\section{Introduction}

Understanding reality is intrinsically bounded and affected by the action of perceiving it. Apart from the uncertainty and error committed and introduced, an interesting point of this step is that not everything is perceivable and hence all perceived objects are built by perceivable bricks which brings granularity into the system.

Let us illustrate the fact that not everything is perceivable. If, for instance, we think of the human eye as a sensor of outside reality we can see that there is a bound on the size of objects that can be seen (very tiny things are not perceived by the human eye), very similar colours cannot be distinguished, rays with wavelength outside the observable interval are not seeable, etcetera.

Further, it is interesting to observe that the perceivable bricks are intrinsically fuzzy. If we take again the human eye as an example, it is easy to imagine a gradation of colours such that in every step the colour is slightly changed (such that this change is not perceived) but the initial and final colour are clearly dissimilar.

According to Zadeh, granularity is one of the basic concepts that underlie human cognition [24] and the elements within a granule "have to be dealt with as a whole rather then individually" [23].

"Informally, granulation of an object A results in a collection of granules of A, with a granule being a clump of objects (or points) which are drawn together by indistinguishability, similarity, proximity or functionality" [24].

Bringing this discussion to mathematical fuzzy logic, we can model a perception of reality as a pair $(X, E)$ where $X$ is a set corresponding to outside reality and $E$ is a relation between the elements of $X$ that "identifies" objects and it is subjective as it is given by the limitations of the sensor.

If we model this relation $E$ by an indistinguishability operator (fuzzy equivalence relation), then the observable sets or granules of $X$ correspond to the extensional fuzzy subsets related to $E$. Hence, extensional sets are exactly these fuzzy bricks that build perceived objects. In other words, taking $E$ into account arbitrary fuzzy subsets cannot be perceived; so they must be replaced by extensional ones. 
The question that arises and motivates this paper is how can we approximate an arbitrary object of outside reality by perceivable reality in the way that less information is lost. In mathematical terms: How can an arbitrary fuzzy subset be approximated by a similar extensional one as close as possible to it.

In the literature this problem has not been faced but indirectly and the main results found on this topic have been the construction of two operators $\phi_{E}[11]$ and $\psi_{E}[5]$ that given a fuzzy subset $\mu$ provide the lowest extensional fuzzy subset containing $\mu$ and the biggest extensional fuzzy subset that contains $\mu$ respectively. However, in general there is no guarantee that there are no extensional sets "in between" that approximate $\mu$ better. This means that it is possible that the fuzzy extensional subset that best approximates $\mu$ is neither containing nor contained in $\mu$.

In this work three methods for finding better approximations of fuzzy subsets by extensional ones are proposed. The first two methods are based on operations with the operators $\phi$ and $\psi$ but their nature are essentially different: the first one is based on finding an adequate mean between these operators while the second one uses the application of powers or homotocies with respect to the t-norm. The third one will be based on quadratic programming.

These results work only for continuous Archimedean t-norms but do not work for the Minimum t-norm. This case will be studied separately and an algorithm to find good extensional approximations of arbitrary fuzzy subsets will be provided.

Finally all these ideas and methods will be illustrated in a complete example.

This work is structured as follows:

In Section 2 the preliminaries to this work will be provided. The definitions of indistinguishability operator and extensional fuzzy subset will be recalled, and the main properties and results this work lies upon will be given.

In Section 3 it will be shown how natural means can be used in order to find good approximations of arbitrary fuzzy subsets by extensional ones. Explicit formulas will be given for the Euckasievicz t-norm and the Product one over a finite set $X$.

Section 4 will be devoted to deriving a different method based on powers 
to find other approximations. As it was done in the previous section, formulas will be derived for the two main continuous Archimedean t-norms and a finite set $X$.

Section 5 will show another approach to find approximations of fuzzy subsets by extensional ones using numerical methods to solve a Quadratic Programming problem.

In Section 6 we will show how the results and formulas found in sections 3 and 4 can be derived as well if we let the set $X$ to be non-finite.

In Section 7 a method to approximate fuzzy subsets by extensional ones when $E$ is a min-indistinguishability operator will be derived. An explicit algorithm will be proposed and the solution will be given by the solution of a Quadratic Programming problem.

Section 8 will provide an example over a given set $X$ and indistinguishability operator $E$ where all the different approximations will be computed. It will be shown how the results found improve the upper and lower approximations obtained with the operators $\phi$ and $\psi$ and a discussion and comparison of the results will be given.

Finally, the concluding remarks of this work will be found in Section 9 .

\section{Indistinguishability Operators and Exten- sional Sets}

In this section the main concepts and results used in this work will be given. The definition of indistinguishability operator will be recalled as well as the main properties of the extensional fuzzy subsets related to an indistinguishability operator.

First of all let us recall the well known Ling's Theorem which introduces the concept of additive generator $t$ of a continuous Archimedean t-norm. Additive generators will prove to be very useful further in this work.

Theorem 2.1. [15] A continuous t-norm $T$ is Archimedean if and only if there exists a continuous and strictly decreasing function $t:[0,1] \rightarrow[0, \infty]$ with $t(1)=0$ such that

$$
T(x, y)=t^{[-1]}(t(x)+t(y))
$$


where $t^{[-1]}$ is the pseudo inverse of $t$ defined by

$$
t^{[-1]}(x)= \begin{cases}1 & \text { if } x \leq 0 \\ t^{-1}(x) & \text { if } 0 \leq x \leq t(0) \\ 0 & \text { if } t(0) \leq x\end{cases}
$$

The function $t$ will be called an additive generator of the t-norm and two generators of the t-norm $T$ differ only by a positive multiplicative constant.

If $T=\mathrm{E}$ is the Eukasievicz t-norm, then an additive generator is $t(x)=$ $1-x$.

If $T=\Pi$ is the Product t-norm, then $t(x)=-\log (x)$.

Definition 2.2. Let $T$ be a $t$-norm.

- The residuation $\vec{T}$ of $T$ is defined for all $x, y \in[0,1]$ by

$$
\vec{T}(x \mid y)=\sup \{\alpha \in[0,1] \mid T(\alpha, x) \leq y\} .
$$

- The birresiduation $\overleftrightarrow{T}$ of $T$ is defined for all $x, y \in[0,1]$ by

$$
\overleftrightarrow{T}(x, y)=\min \{\vec{T}(x \mid y), \vec{T}(y \mid x)\}=T(\vec{T}(x \mid y), \vec{T}(y \mid x))
$$

When the t-norm $T$ is continuous Archimedean, these operations can be rewritten in terms of the additive generator $t$.

Proposition 2.3. Let $T$ be a continuous t-norm generated by an additive generator $t$. Then:

- $T(x, y)=t^{[-1]}(t(x)+t(y))$

- $\vec{T}(x \mid y)=t^{[-1]}(t(y)-t(x))$

- $\overleftrightarrow{T}(x, y)=t^{[-1]}(|t(x)-t(y)|)$

Indistinguishability operators are the fuzzification of classical equivalence relations and model the intuitive idea of "similarity" between objects. For a more detailed explanation on this operators readers are referred to [4], [19]. 
Definition 2.4. Let $T$ be a t-norm. A fuzzy relation $E$ on a set $X$ is a $T$-indistinguishability operator if and only if for all $x, y, z \in X$

a) $E(x, x)=1$ (Reflexivity)

b) $E(x, y)=E(y, x)$ (Symmetry)

c) $T(E(x, y), E(y, z)) \leq E(x, z)$ (T-transitivity).

Indistinguishability operators can be generated in multiple ways. One of them is considering the indistinguishability operator generated by a fuzzy subset $\mu$.

Proposition 2.5. Let $X$ be a set, $T$ a continuous t-norm and $\mu$ a fuzzy subset of $X$. The fuzzy relation $E_{\mu}$ on $X$ defined by

$$
E_{\mu}(x, y)=\overleftrightarrow{T}(\mu(x), \mu(y))
$$

for all $x, y \in X$ is a $T$-indistinguishability operator.

Whereas indistinguishability operators represent the fuzzification of equivalence relations, extensional fuzzy subsets play the role of fuzzy equivalence classes altogether with their intersections and unions. Extensional fuzzy subsets are a key concept in the comprehension of the universe of discourse $X$ under the effect of an indistinguishability operator $E$ as they correspond with the observable sets or granules of $X$.

Definition 2.6. Let $X$ be a set and $E$ a T-indistinguishability operator on $X$. A fuzzy subset $\mu$ of $X$ is called extensional with respect to $E$ if and only if:

$$
\forall x, y \in X \quad T(E(x, y), \mu(y)) \leq \mu(x) .
$$

We will denote $H_{E}$ the set of all extensional fuzzy subsets of $X$ with respect to $E$.

Extensional fuzzy subsets have been widely studied in the literature [7], [11], [12]. Below we recall one of their most interesting characterizations.

Corollary 2.7. [11] Let $X$ be a set, E a T-indistinguishability operator on $X$ and $\mu$ a fuzzy subset of $X$. Then:

$$
\mu \in H_{E} \Leftrightarrow E_{\mu} \geq E .
$$


If the t-norm $T$ is continuous Archimedean then the condition of extensionality can be rewritten in terms of additive generators. This result will be recalled several times along this paper.

Lemma 2.8. Let $E$ be a $T$-indistinguishability operator on a set $X . \mu \in H_{E}$ if and only if $\forall x, y \in X$ :

$$
t(E(x, y))+t(\mu(y)) \geq t(\mu(x)) .
$$

Proof.

$\mu \in H_{E} \Leftrightarrow T(E(x, y), \mu(y)) \leq \mu(x) \Leftrightarrow t^{-1}(t(E(x, y))+t(\mu(y))) \leq \mu(x)$.

And as $t$ is a monotone decreasing function this is equivalent to

$$
t(E(x, y))+t(\mu(y)) \geq t(\mu(x)) .
$$

\section{Approximation using Natural Weighted Means}

In this section we will propose a method to approximate an arbitrary fuzzy subset by an extensional one. First we will introduce two approximation operators, $\phi_{E}(\mu)$ and $\psi_{E}(\mu)$, that provide the best upper and lower approximation respectively by extensional fuzzy subsets of $\mu$. The method will consist in computing an adequate weight in order to minimize an error function between $\mu$ and the natural weighted mean of $\phi_{E}(\mu)$ and $\psi_{E}(\mu)$.

Definition 3.1. Let $X$ be a set and $E$ a $T$-indistinguishability operator on $X$. The maps $\phi_{E}:[0,1]^{X} \rightarrow[0,1]^{X}$ and $\psi_{E}:[0,1]^{X} \rightarrow[0,1]^{X}$ are defined $\forall x \in X$ by:

$$
\begin{aligned}
& \phi_{E}(\mu)(x)=\sup _{y \in X} T(E(x, y), \mu(y)), \\
& \psi_{E}(\mu)(x)=\inf _{y \in X} \vec{T}(E(x, y) \mid \mu(y)) .
\end{aligned}
$$

$\phi_{E}(\mu)$ is the smallest extensional fuzzy subset greater than or equal to $\mu$; hence it is its best upper approximation by extensional fuzzy subsets. Analogously, $\psi_{E}(\mu)$ provides the best approximation by extensional fuzzy subsets smaller than or equal to $\mu$. From a topological viewpoint these operators can be seen as closure and interior operators on the set $[0,1]^{X}[11]$. It is remarkable that these operators also appear in a natural way in fields such as fuzzy 
rough sets [18], fuzzy modal logic [6], [5], fuzzy mathematical morphology [8] and fuzzy contexts [3] among many others.

In Section 8 an example of these operators is provided for a given indistinguishability $E$ and fuzzy subset $\mu$.

Though $\phi_{E}(\mu)$ and $\psi_{E}(\mu)$ provide extensional fuzzy subsets that approximate $\mu$ there is no guarantee in general that there are no better approximations of $\mu$ by extensional fuzzy subsets. The aim of this paper is to provide methods to find approximations such that the error made is lower.

Definition 3.2. [1] Let $t:[0,1] \rightarrow[-\infty, \infty]$ be a non-increasing monotonic map, $x, y \in[0,1]$ and $r \in[0,1]$. The weighted quasi-arithmetic mean $m_{t}$ of $x$ and $y$ is defined as:

$$
m_{t}^{r}(x, y)=t^{-1}(r \cdot t(x)+(1-r) \cdot t(y))
$$

$m_{t}$ is continuous if and only if $\{-\infty, \infty\} \nsubseteq \operatorname{Im}(t)$, being $\operatorname{Im}(t)$ the image set of the map $t$.

There is a bijection between the set of continuous Archimedean t-norms and the set of quasi-arithmetic means by taking as map the additive generator $t$ of the t-norm [14]. Under this interpretation in the literature quasiarithmetic means are sometimes called natural means [17], as we will recall them from now on.

We want to approximate $\mu$ by $m_{t}^{r}\left(\phi_{E}(\mu), \psi_{E}(\mu)\right)$. Below we prove that this mean is extensional for any value of $r$.

Proposition 3.3. Let $X$ be a set and $\mu, \nu$ extensional fuzzy subsets of $X$ with respect to an indistinguishability operator $E$ on $X$. Then:

$$
m^{r}(\mu, \nu) \in H_{E}
$$

Proof. We have to prove that

$$
T\left(E(x, y), m^{r}(\mu, \nu)(y) \leq m^{r}(\mu, \nu)(x) .\right.
$$

Expanding $T$ using $t$ this is equivalent to

$$
t(E(x, y))+r \cdot t(\mu)(y))+(1-r) \cdot t(\nu)(y)) \leq r \cdot t((\mu)(x))+(1-r) \cdot t((\nu)(x))
$$

and we can rewrite this expression as: 


$$
\begin{gathered}
r \cdot t(E(x, y))+r \cdot t\left(\phi_{E}(\mu)(y)\right)+(1-r) \cdot t(E(x, y))+(1-r) \cdot t\left(\psi_{E}(\mu)(y)\right) \\
r \cdot t\left(\phi_{E}(\mu)(x)\right)+(1-r) \cdot t\left(\psi_{E}(\mu)(x)\right)
\end{gathered}
$$

which is true because $\mu$ and $\nu$ are extensional fuzzy subsets and due to the characterization of extensional sets given in Lemma 2.8 .

Corollary 3.4. Let $\mu$ be a fuzzy subset on a set $X$ and $E$ an indistinguishability operator. Then:

$$
m^{r}\left(\phi_{E}(\mu), \psi_{E}(\mu)\right) \in H_{E} .
$$

It is straightforward that for the limit values $r=0,1$ this mean is equal to $\phi_{E}(\mu)$ and $\psi_{E}(\mu)$ respectively. The question that arises here is for what value of $r$ the error made in this approximation is lower. In mathematical terms, this problem reduces to finding the minimum value of the following function:

$$
F(r)=\left\|\mu-m^{r}\left(\phi_{E}, \psi_{E}\right)\right\|
$$

Considering the Euclidean distance, without loss of generalization, minimizing the previous expression is equivalent to minimize the square of the norm.

$$
F(r)=\left\|\mu-m^{r}\left(\phi_{E}, \psi_{E}\right)\right\|^{2}
$$

For the Eukasievicz t-norm the result below provides an explicit formula to find this optimal weight $r$.

Theorem 3.5. Let $\mu$ be a fuzzy subset of a finite set $X=\left\{x_{1}, \ldots, x_{n}\right\}$ and $T=$ $E$ the Eukasiewicz t-norm. Then the expression $F(r)=\left\|\mu-m^{r}\left(\phi_{E}, \psi_{E}\right)\right\|^{2}$ is minimized when:

$r=\frac{\sum \mu\left(x_{i}\right) \phi_{E}(\mu)\left(x_{i}\right)-\sum \mu\left(x_{i}\right) \psi_{E}(\mu)\left(x_{i}\right)-\sum \phi_{E}(\mu)\left(x_{i}\right) \psi_{E}(\mu)\left(x_{i}\right)+\sum\left(\psi_{E}(\mu)\left(x_{i}\right)\right)^{2}}{\sum\left(\phi_{E}(\mu)\left(x_{i}\right)\right)^{2}+\sum\left(\psi_{E}(\mu)\left(x_{i}\right)\right)^{2}-2 \sum \phi_{E}(\mu)\left(x_{i}\right) \psi(\mu)\left(x_{i}\right)}$

Proof. In order to simplify the notation we will denote $\mu_{i}=\mu\left(x_{i}\right), \phi_{i}=$ $\phi_{E}(\mu)\left(x_{i}\right)$ and $\psi_{i}=\psi_{E}(\mu)\left(x_{i}\right)$.

As $T=\mathrm{E}$ we can take as additive generator $t(x)=1-x$. Expanding $F(r)$ we have:

$$
F(r)=\left(\mu_{1}-r \cdot \phi_{1}+r \cdot \psi_{1}-\psi_{1}\right)^{2}+\cdots+\left(\mu_{n}-r \cdot \phi_{n}+r \cdot \psi_{n}-\psi_{n}\right)^{2} .
$$


The derivative of $F$ is:

$$
\begin{aligned}
F^{\prime}(r)= & 2\left(\mu_{1} \cdot \psi_{1}-\mu_{1} \cdot \phi_{1}+r \cdot \phi_{1}^{2}+r \cdot \psi_{1}^{2}-2 r \cdot \phi_{1} \cdot \psi_{1}-\psi_{1}^{2}\right)+\cdots \\
& +2\left(\mu_{n} \cdot \psi_{n}-\mu_{n} \cdot \phi_{n}+r \cdot \phi_{n}^{2}+r \cdot \psi_{n}^{2}-2 r \cdot \phi_{n} \cdot \psi_{n}-\psi_{n}^{2}\right)
\end{aligned}
$$

and the only value of $r$ for which $F^{\prime}(r)=0$ is:

$$
r=\frac{\sum \mu_{i} \cdot \phi_{i}-\sum \mu_{i} \cdot \psi_{i}-\sum \phi_{i} \cdot \psi_{i}+\sum \psi_{i}^{2}}{\sum \phi_{i}^{2}+\sum \psi_{i}^{2}-2 \sum \phi_{i} \cdot \psi_{i}}
$$

From the nature of the problem it is straightforward that this value is a minimum. However it can be checked that $F^{\prime}(0)<0$ and $F^{\prime}(1)>0$. Hence the value found is a global minimum.

If we consider the Product t-norm $T=\Pi$, the expanded expression of $F(r)$ is:

$$
F(r)=\left(\mu_{1}-e^{r \cdot \log \phi_{1}+(1-r) \cdot \log \psi_{1}}\right)^{2}+\cdots\left(\mu_{n}-e^{r \cdot \log \phi_{n}+(1-r) \cdot \log \psi_{n}}\right)^{2}
$$

and the derivative $F^{\prime}(r)$ is

$F^{\prime}(r)=-2\left(\mu_{1}-e^{r \cdot \log \phi_{1}+(1-r) \cdot \log \psi_{1}}\right)\left(-e^{r \cdot \log \phi_{1}+(1-r) \cdot \log \psi_{1}}\right)\left(\log \phi_{1}-\log \psi_{1}\right)+\cdots$

But this expression has no algebraic zero if $n>1$. Thus, the solution must be found using numerical methods. In Section 7 we will illustrate with an example how it can be computed.

\section{Approximation using Powers}

In this section we will provide another method to find an approximation of an arbitrary fuzzy subset $\mu$ by an extensional one. This method will be based on approximating $\mu$ by an adequate power $\psi_{E}(\mu)^{r}$ of its lower approximation operator with respect to the t-norm. It will be shown how, for values $r<1$, the fuzzy subset $\psi_{E}(\mu)^{r}$ is extensional and that a global minimum of the error made can be obtained.

Let us recall the definition of power with respect to a t-norm $T$. 
Definition 4.1. Let $T$ be a t-norm and $n$ a natural number. We will call the $n^{\text {th }}$ power of $X$ with respect to $T$ to:

$$
T^{n}(x)=T(\overbrace{x, x, \ldots, x}^{n}) .
$$

To simplify notation we will denote $T^{n}(x)=x^{n}$.

It is possible to extend this definition to all positive rational numbers as follows.

Definition 4.2. Let $T$ be a t-norm and $n$ a natural number. We will define $x$ to the power of $1 / n$ with respect to $T$ as:

$$
x^{1 / n}=\sup _{z \in[0,1]} T^{n}(z) \leq x
$$

and for $p, q$ natural numbers,

$$
x^{p / q}(x)=\left(x^{1 / q}\right)^{p} .
$$

Passing to the limit it is possible to define $x^{r}$ for all $r \in \mathbb{R}^{+}$for continuous t-norms.

The following result allows us to calculate powers by using an additive generator $t$ of $T$.

Proposition 4.3. Let $T$ be an Archimedean t-norm with additive generator $t$ and $r \in \mathbb{R}^{+}$. Then:

$$
x^{r}=t^{[-1]}(r \cdot t(x))
$$

It is straightforward to observe from the previous proposition that $r \leq$ $s \Rightarrow x^{r} \geq x^{s}$. Besides, the continuity of $t$ assures continuity of powers when we let the exponent vary.

The key idea of this method follows from the next Corollary 4.5.

Proposition 4.4. Let $E$ be an indistinguishability operator of a set $X, \mu$ an extensional fuzzy subset of $E$ and $r \leq 1$. Then

$$
\mu^{r} \in H_{E} .
$$


Proof.

$\mu$ is extensional, and by Lemma $2.8 t(E(x, y)) \geq t(\mu(x))-t(\mu(y))$.

We have to see that $T\left(E(x, y), \mu(y)^{r}\right) \leq \mu(x)^{r}$.

Expanding,

$$
t(E(x, y)+r \cdot t(\mu(x)) \geq r \cdot t(\mu(y))
$$

or, equivalently,

$$
t(E(x, y) \geq r \cdot(t(\mu(x))-t(\mu(y)))
$$

which is true because $r \leq 1$.

Corollary 4.5. $\psi(\mu)^{r}$ is extensional for $r \leq 1$.

The problem of approximating a fuzzy subset $\mu$ by an adequate power $\psi(\mu)^{r}$ reduces then to compute the value of $r$ for which the following function is minimized.

$$
F(r)=\left\|\mu-\psi_{E}(\mu)^{r}\right\|^{2}
$$

For the Eukasievicz t-norm we have the following result.

Theorem 4.6. Let $\mu$ be a fuzzy subset of a finite set $X=\left\{x_{1}, \ldots, x_{n}\right\}$ and $T=E$ the Eukasiewicz t-norm. Then $F(r)=\left\|\mu-\psi_{E}(\mu)^{r}\right\|^{2}$ is minimized when

$$
r=\frac{\sum \mu\left(x_{i}\right)+\sum \psi_{E}(\mu)\left(x_{i}\right)-\sum \mu\left(x_{i}\right) \cdot \psi_{E}(\mu)\left(x_{i}\right)-n}{2 \sum \psi_{E}(\mu)\left(x_{i}\right)-\sum \psi_{E}^{2}(\mu)\left(x_{i}\right)-n}
$$

Proof. In order to simplify the notation we will denote $\mu_{i}=\mu\left(x_{i}\right)$ and $\psi_{i}=$ $\psi(\mu)\left(x_{i}\right)$.

We can take as additive generator $t(x)=1-x$.

$$
F(r)=\left(\mu_{1}-1+r-r \cdot \psi_{1}\right)^{2}+\cdots+\left(\mu_{n}-1+r-r \cdot \psi_{n}\right)^{2} .
$$

The derivative is

$$
F^{\prime}(r)=2\left(\mu_{1}+\psi_{1}-\mu_{1} \cdot \psi_{1}+\cdots \mu_{n}+\psi_{n}-\mu_{n} \cdot \psi_{n}-n\right)-2 r\left(2 \psi_{1}-\psi_{1}^{2}+\cdots+2 \psi_{n}-\psi_{n}^{2}-n\right)
$$

and the only value of $r$ for which $F^{\prime}(r)=0$ is

$$
r=\frac{\sum \mu_{i}+\sum \psi_{i}-\sum \mu_{i} \cdot \psi_{i}-n}{2 \sum \psi_{i}-\sum \psi_{i}^{2}-n}
$$


If we consider the Product t-norm $T=\Pi$, we have the same situation of the previous section. The function to be minimized is

$$
F(r)=\left\|\mu-\psi_{E}^{r}(\mu)\right\|^{2}=\left(\mu_{1}-e^{r \cdot \log \left(\psi_{1}\right)}\right)^{2}+\cdots+\left(\mu_{n}-e^{r \cdot \log \left(\psi_{n}\right)}\right)^{2} .
$$

If we compute the derivative we have

$$
F^{\prime}(r)=-2 \sum \log \left(\psi_{i}\right) \cdot\left(\mu_{i}-e^{r \cdot \log \left(\psi_{i}\right)}\right)
$$

and if $n>1$ there is no algebraic zero of this expression and thus numerical methods have to be used to find the solution of $F^{\prime}(r)=0$.

Finally, given the duality between upper and lower approximations, $\phi_{E}$ and $\psi_{E}$, it would be expectable to find an analogous method to find an approximation based on powers of $\phi_{E}(\mu)$. But this is not possible.

Indeed, $\phi_{E}(\mu)$ is an upper approximation of $\mu$, thus better approximations $\phi(\mu)^{r}$ would be found for values $r>1$. However, if $\nu$ is an extensional fuzzy subset, for values of $r$ greater than $1, \nu^{r}$ is not extensional in general.

Example 4.7. Let $T=E$ be the Eukasiewicz t-norm, E the E-indistinguishability operator with matrix

$$
\begin{gathered}
E=\left(\begin{array}{lllll}
1 & 0.8 & 0.7 & 0.3 & 0.2 \\
0.8 & 1 & 0.7 & 0.3 & 0.2 \\
0.7 & 0.7 & 1 & 0.3 & 0.2 \\
0.3 & 0.3 & 0.3 & 1 & 0.2 \\
0.2 & 0.2 & 0.2 & 0.2 & 1
\end{array}\right) \\
\quad(x)=\left(\begin{array}{l}
0.9 \\
0.7 \\
0.6 \\
0.8 \\
0.3
\end{array}\right)
\end{gathered}
$$

and $r=2$. Then

$$
\nu^{2}(x)=\left(\begin{array}{l}
0.8 \\
0.4 \\
0.2 \\
0.6 \\
0
\end{array}\right)
$$


and the condition of extensionality is not fulfilled for $\nu^{2}$ :

$$
T\left(E\left(x_{1}, x_{2}\right), \nu^{2}\left(x_{1}\right)\right)=0.8+0.8-1=0.6>0.4=\nu^{2}\left(x_{2}\right) .
$$

Hence, $\nu^{r}(r>1)$ is not extensional in general.

\section{Approximation using Quadratic Program- ming}

In this section a third method to approximate a fuzzy subset by an extensional one based on solving a Quadratic Programming (QP) problem will be developed. The aim will be to look for the fuzzy subset that better approximates a given subset $\mu$ restricting the search to the region of extensional fuzzy subsets, which for the Eukasiewicz ant Product t-norms will be characterized by a system of linear inequalities.

At the end of the section a brief theoretical comparison between the three proposed methods will be provided, in terms of quality of the solution, and applicability and complexity of the methods.

We will assume that $X=\left\{x_{1}, \ldots, x_{n}\right\}$ is a finite set.

Let us denote by $\mu$ an arbitrary fuzzy subset and $\sigma$ an arbitrary extensional fuzzy subset with respect to a $T$-indistinguishability operator $E$ on $X$. The objective function to be minimized is

$$
F=\|\mu-\sigma\|^{2}
$$

The region of the space in which the solution has to be found is bounded by the conditions that ensure the extensionality of $\sigma$. Assuming $T$ to be continuous Archimedean with additive generator $t$, following from Lemma 2.8 we have that $\sigma$ is extensional if and only if verifies

$$
t\left(E\left(x_{i}, x_{j}\right)+t\left(\sigma\left(x_{j}\right)\right) \geq t\left(\sigma\left(x_{i}\right)\right) \quad \forall i, j=1, \ldots, n .\right.
$$

If $T=\mathrm{E}$ is the Łukasiewiz t-norm, then we can take as additive generator $t(x)=1-x$ and these inequalities become

$$
\sigma\left(x_{i}\right)-\sigma\left(x_{j}\right) \geq E\left(x_{i}, x_{j}\right)-1 \quad \forall i, j=1, \ldots, n .
$$

Analogously, if $T=\Pi$ is the Product t-norm, these conditions are 


$$
\sigma\left(x_{i}\right) \geq E\left(x_{i}, x_{j}\right) \cdot \sigma\left(x_{j}\right) \quad \forall i, j=1, \ldots, n .
$$

In both cases this is a system of $n$ unknowns and $\left(\begin{array}{l}n \\ 2\end{array}\right)$ inequalities (excluding the trivial cases $i=j$ ).

In order to ensure that the usage of numerical techniques converges we have to verify that the search space is compact in $\mathbb{R}^{n}$. However this is trivial given that $\sigma\left(x_{i}\right) \leq 1 \forall i=1, \ldots, n$ and hence the region is closed and bounded.

The best approximation of $\mu$ by an extensional fuzzy subset (i.e. the one minimizing $F$ ) is then the solution of a Qadratic Programming problem and several algorithms to solve it can be applied [2].

Let us point out some final considerations about this method in comparison with the ones provided in Sections 3 and 4.

The previous two methods assumed some kind of structure on the target output, being either a natural weighted mean or a power of previous extensional fuzzy subsets. This third method searches for the best approximation in the space of all extensional fuzzy subsets. Hence the output will be always better than or equal to the mean-based and power-based method one.

However, there are two main cons to this method against the others.

Firstly, in Section 6 we will show how the first two methods can be extended to non-finite sets, whereas the QP-based one only works on finite sets. If $X$ was a non-finite set then the system of inequalities to characterize the extensional region would be infinite and the method would not be applicable.

Besides, the complexity of solving a QP problem is $O(n !)$ while the meanbased and power-based methods are polynomial. Hence, for large cardinalities of $X$ the QP-based method becomes unaffordable.

\section{Approximation on a non-finite Set}

In this section the methods proposed in Sections 3 and 4 will be extended if we let $X$ to be a non-finite set.

The definition of the operators $\phi_{E}$ and $\psi_{E}$ is independent of the cardinality of $X$ and thus if the set $X$ is non-finite the approaches of the mean-based and power-based methods can be constructed in a similar way. If we de- 
note by $\sigma$ an extensional set (either $m^{r}\left(\phi_{E}(\mu), \psi_{E}(\mu)\right)$ or $\psi_{E}^{r}(\mu)$ ), the error function becomes

$$
F=\|\mu-\sigma\|^{2}=\int_{X}(\mu(x)-\sigma(x))^{2} d x .
$$

The following result proves that this function is well defined if $X$ is a measurable space.

Proposition 6.1. Let $X$ be a measurable non-finite set and $E$ a measurable indistinguishability operator on $E$. Then $E$ is Lebesgue-integrable.

Proof. A bounded and measurable function is Lebesgue integrable [21]

Corollary 6.2. Let $\mu$ be a measurable fuzzy subset of $X$. Then $\phi_{E}, \psi_{E}$ are Lebesgue-integrable.

Proof. The supremum/infimum and composition of Lebesgue integrable functions is Lebesgue integrable [21]

Hence, either the mean-based method and the power-based method can be constructed for measurable non-finite sets. The results below show that the formulas obtained for the Eukasievicz t-norm are analogous to the ones found in Sections 3 and 4 replacing sums by integrals.

Theorem 6.3. Let $\mu$ be a measurable fuzzy subset of a measurable non-finite set $X$ with measure $m(X)$ and $T=E$ the Eukasiewicz t-norm. Then the expression $F(r)=\left\|\mu-m^{r}(\phi, \psi)\right\|^{2}$ is minimized when

$$
r=\frac{\int_{X} \mu\left(x_{i}\right) \phi_{E}(\mu)\left(x_{i}\right) d x-\int_{X} \mu\left(x_{i}\right) \psi_{E}(\mu)\left(x_{i}\right) d x-\int_{X} \phi_{E}(\mu)\left(x_{i}\right) \psi_{E}(\mu)\left(x_{i}\right) d x+\int_{X}\left(\psi_{E}(\mu)\left(x_{i}\right)\right)^{2}}{\int_{X}\left(\phi_{E}(\mu)\left(x_{i}\right)\right)^{2} d x+\int_{X}\left(\psi_{E}(\mu)\left(x_{i}\right)\right)^{2} d x-2 \int_{X} \phi_{E}(\mu)\left(x_{i}\right) \psi(\mu)\left(x_{i}\right) d x}
$$

Theorem 6.4. Let $\mu$ be a measurable fuzzy subset of an non-finite set $X$ with measure $m(X)<\infty$ and $T=E$ the Eukasiewicz t-norm. Then the expression $\left.F(r)=\| \mu-\psi_{E}^{r}\right) \|^{2}$ is minimized when:

$$
r=\frac{\int \mu\left(x_{i}\right) d x+\int \psi_{E}(\mu)\left(x_{i}\right) d x-\int \mu\left(x_{i}\right) \cdot \psi_{E}(\mu)\left(x_{i}\right) d x-m(X)}{2 \int \psi_{E}(\mu)\left(x_{i}\right) d x-\sum \psi_{E}^{2}(\mu)\left(x_{i}\right) d x-m(X)} .
$$


The proof for these results is analogous to the ones in Sections 3 and 4 and based on the following lemma.

Lemma 6.5. (Leibniz's rule)[9] Let $f(x, r)$ be an integrable function derivable with respect to the second variable. Then

$$
\frac{\partial}{\partial r} \int f(x, r) d x=\int \frac{\partial f}{\partial r}(x, r) d x .
$$

A sketch of the proof goes as follows:

The function to be minimized is

$$
F(r)=\|\mu-\sigma\|^{2}=\int_{X}(\mu(x)-\sigma(r)(x))^{2} d x
$$

Then,

$$
F^{\prime}(r)=\left(\int_{X}(\mu(x)-\sigma(r)(x))^{2} d x\right)^{\prime}
$$

which, due to Lemma 6.5, is equivalent to

$$
F^{\prime}(r)=\int_{X} \frac{\partial}{\partial r}(\mu(x)-\sigma(r)(x))^{2} d x
$$

and the rest of the argument is analogous to the ones in Sections 3 and 4.

\section{Approximation for the Minimum t-norm}

The methods developed in the previous sections do not work for the Minimum t-norm. In this section we will propose a method to obtain good approximations of fuzzy subsets by extensional ones with respect to a given min-indistinguishability operator.

The key of this method comes after the following Lemma.

Lemma 7.1. Let $X=\left\{x_{1}, \ldots, x_{n}\right\}$ be a finite set, $E$ a min-indistinguishability operator and $\sigma$ an extensional fuzzy subset. Consider the subset $A_{\sigma} \subseteq[0,1]$ consisting of all the images of $E$ and all the images of $\sigma$. Then the fuzzy subsets $\sigma^{\prime}$ such that there is a non-decreasing map $f: A_{\sigma} \rightarrow A_{\sigma^{\prime}}$ with $f(a)=$ a $\forall a \in \operatorname{Im}(E)$ are extensional with respect to $E$. 
Proof. Extensionality with respect to the Minimum t-norm only depends on the ordering of the values of $\mu$ and the entries of $E$. Hence,

$$
\min \left(E\left(x_{i}, x_{j}\right), \sigma\left(x_{j}\right)\right) \leq \sigma\left(x_{i}\right)
$$

implies

$$
\min \left(E\left(x_{i}, x_{j}\right), \sigma^{\prime}\left(x_{j}\right)\right) \leq \sigma^{\prime}\left(x_{i}\right)
$$

In order to find a good approximation of a fuzzy subset $\mu$ by an extensional one with respect to $E$, we can calculate its upper (or lower) approximation and find $A_{\phi_{E}(\mu)}$ (respectively $A_{\psi_{E}(\mu)}$ ) and adjust its values according to Lemma 7.1 in order to minimize the distance with $\mu$. This is a Quadratic Programming problem described in the following algorithm.

Algorithm 7.2. Let $E$ be a min-indistinguishability operator on a finite set $X=\left\{x_{1}, \ldots, x_{n}\right\}$ and $\mu$ a fuzzy subset.

1. Calculate $\phi_{E}(\mu)$ (or $\left.\psi_{E}(\mu)\right)$ and find $A_{\phi_{E}(\mu)}\left(\right.$ or $\left.A_{\psi_{E}(\mu)}\right)$.

2. Consider the restrictions of a fuzzy subset $\sigma(x)=\left(\sigma_{1}, \ldots, \sigma_{n}\right)$ according to Lemma 7.1.

3. Minimize $F=\|\mu-\sigma\|$ with the previous restrictions.

Let us illustrate this algorithm with an example.

Example 7.3. Consider the following min-indistinguishability operator

$$
E=\left(\begin{array}{llll}
1 & 0.7 & 0.4 & 0.1 \\
0.7 & 1 & 0.4 & 0.1 \\
0.4 & 0.4 & 1 & 0.1 \\
0.1 & 0.1 & 0.1 & 1
\end{array}\right)
$$

and the fuzzy subset

$$
\mu(x)=\left(\begin{array}{c}
0.3 \\
0.5 \\
0.9 \\
0.2
\end{array}\right)
$$


which is not extensional: $\min \left\{E\left(x_{1}, x_{2}\right), \mu\left(x_{2}\right)\right\}=0.5>0.3=\mu\left(x_{1}\right)$.

Then

$$
\phi_{E}(\mu)(x)=\left(\begin{array}{c}
0.5 \\
0.5 \\
0.9 \\
0.2
\end{array}\right) \quad \psi_{E}(\mu)(x)=\left(\begin{array}{c}
0.3 \\
0.3 \\
0.3 \\
0.3
\end{array}\right)
$$

The corresponding sets $A_{\phi_{E}(\mu)}$ and $A_{\psi_{E}(\mu)}$ are

$$
\begin{gathered}
A_{\phi_{E}(\mu)}=\{1,0.9,0.7,0.5,0.4,0.2,0.1\} \\
A_{\psi_{E}(\mu)}=\{1,0.7,0.4,0.3,0.1\}
\end{gathered}
$$

Using as initial extensional input in Algorithm 7.2 the extensional fuzzy subset $\phi_{E}(\mu)$ the quadratic problem to solve is:

$$
F=\left(0.3-\sigma_{1}\right)^{2}+\left(0.5-\sigma_{2}\right)^{2}+\left(0.9-\sigma_{3}\right)^{2}+\left(0.2-\sigma_{4}\right)^{2}
$$

subject to

$$
\begin{gathered}
0.4 \leq \sigma_{1}=\sigma_{2} \leq 0.7 \\
0.7 \leq \sigma_{3} \leq 1 \\
0.1 \leq \sigma_{4} \leq 0.4
\end{gathered}
$$

And the solution is

$$
\sigma_{\phi}(x)=\left(\begin{array}{c}
0.4 \\
0.4 \\
0.9 \\
0.2
\end{array}\right)
$$

Using $\psi_{E}(\mu)$ the restrictions are

$$
0.1 \leq \sigma_{1}=\sigma_{2}=\sigma_{3}=\sigma_{4} \leq 0.4
$$

And the solution is 


$$
\sigma_{\psi}(x)=\left(\begin{array}{c}
0.35 \\
0.35 \\
0.35 \\
0.35
\end{array}\right)
$$

Finally, the error committed by these approximations is

$$
\begin{aligned}
& d\left(\sigma_{\phi}, \mu\right)=0.141421 \\
& d\left(\sigma_{\psi}, \mu\right)=0.591608
\end{aligned}
$$

while

$$
\begin{gathered}
d\left(\phi_{E}(\mu), \mu\right)=0.2 \\
d\left(\psi_{E}(\mu), \mu\right)=0.640312 .
\end{gathered}
$$

\section{Example}

In this section we will apply the methods developed in this paper to approximate a fuzzy subset by an extensional one. We will calculate among a fixed indistinguishability operator what is the extensional subset provided by each of the methods and compare the error made in the approximation, either in the finite and non-finite case for the Archimedean basic t-norms $T=\mathrm{E}$ and $T=\Pi$.

We will use the formulas derived in Sections 3, 4, 5 and 6 for the Eukasievicz t-norm, and numerical methods for the Product t-norm.

Let us consider the fuzzy relation

$$
E=\left(\begin{array}{lllll}
1 & 0.8 & 0.7 & 0.3 & 0.2 \\
0.8 & 1 & 0.7 & 0.3 & 0.2 \\
0.7 & 0.7 & 1 & 0.3 & 0.2 \\
0.3 & 0.3 & 0.3 & 1 & 0.2 \\
0.2 & 0.2 & 0.2 & 0.2 & 1
\end{array}\right)
$$

which is a min-indistinguishability operator and therefore a $T$-indistinguishability operator for any t-norm, and the following fuzzy subset. 


$$
\mu(x)=\left(\begin{array}{l}
0.9 \\
0.5 \\
0.1 \\
0.8 \\
0.3
\end{array}\right)
$$

It is straightforward to observe that $\mu$ is not extensional with respect to $E$ for $T=\mathrm{E}$ :

$$
T\left(E\left(x_{1}, x_{2}\right), \mu\left(x_{1}\right)=0.8+0.9-1=0.7>0.5=\mu\left(x_{2}\right) .\right.
$$

As $T=\mathrm{E} \leq \Pi \leq \min , \mu$ is also not extensional for $\Pi$ and min.

Let us study first the case $T=\mathrm{E}$. Then:

$$
\phi_{E}(\mu)(x)=\left(\begin{array}{c}
0.9 \\
0.7 \\
0.6 \\
0.8 \\
0.3
\end{array}\right) \quad \psi_{E}(\mu)(x)=\left(\begin{array}{l}
0.4 \\
0.4 \\
0.1 \\
0.8 \\
0.3
\end{array}\right) .
$$

The first method proposed in this paper was based on finding the weight $r$ for which the error committed in the approximation of $\mu$ by the natural weighted mean of $\phi_{E}$ and $\psi_{E}$ was minimum. Let $\sigma_{m}$ be this extensional fuzzy subset.

$$
\sigma_{m}=m_{t}^{r}\left(\phi_{E}(\mu), \psi_{E}(\mu)\right)
$$

According to the formula found on Theorem 3.5 we obtain $r=0.116463$. Hence,

$$
\sigma_{m}(x)=\left(\begin{array}{l}
0.637288 \\
0.542373 \\
0.337288 \\
0.8 \\
0.3
\end{array}\right)
$$

The second method proposed in this paper consisted in computing the best exponent $r$ for which the distance between $\mu$ and the extensional fuzzy subset $\psi^{r}(\mu)$ was minimum. 


$$
\sigma_{p}=\psi^{r}(\mu)
$$

According to the formula provided in Theorem 4.6 we obtain $r=0.825243$. Then,

$$
\sigma_{p}(x)=\left(\begin{array}{c}
0.504854 \\
0.504854 \\
0.257282 \\
0.834951 \\
0.422330
\end{array}\right)
$$

Finally, the third method was based on solving a quadratic programming problem. Applied to our particular example, the extensional fuzzy subset solution to the problem is:

$$
\sigma_{Q P}(x)=\left(\begin{array}{l}
0.65 \\
0.5 \\
0.35 \\
0.8 \\
0.3
\end{array}\right)
$$

Let us compare the error committed by each of these approximations. Considering the Euclidean distance, the error made in the approximation by each of the methods is

$$
\begin{gathered}
d\left(\sigma_{m}, \mu\right)=0.341208 \\
d\left(\sigma_{p}, \mu\right)=0.475746 \\
d\left(\sigma_{Q P}, \mu\right)=0.328105
\end{gathered}
$$

while

$$
\begin{aligned}
d\left(\phi_{E}(\mu), \mu\right) & =0.538516 \\
d\left(\psi_{E}(\mu), \mu\right) & =0.509902 .
\end{aligned}
$$

Let us study now the results for the Product t-norm $T=\Pi$. Then 


$$
\phi_{E}(\mu)(x)=\left(\begin{array}{l}
0.9 \\
0.72 \\
0.63 \\
0.8 \\
0.3
\end{array}\right) \quad \psi_{E}(\mu)(x)=\left(\begin{array}{l}
0.142857 \\
0.142857 \\
0.1 \\
0.333333 \\
0.3
\end{array}\right)
$$

Computing the best weight in the mean-based method we obtain $r=$ 0.834 , then

$$
\sigma_{m}(x)=\left(\begin{array}{l}
0.774314 \\
0.624194 \\
0.54202 \\
0.722533 \\
0.3
\end{array}\right)
$$

For the power-based method the minimum is reached when $r=0.394625$. Then the extensional fuzzy subset $\sigma_{p}$ is

$$
\sigma_{p}(x)=\left(\begin{array}{l}
0.463984 \\
0.463984 \\
0.403065 \\
0.64821 \\
0.621812
\end{array}\right)
$$

Finally, with the QP-based method we obtain the following extensional fuzzy subset:

$$
\sigma_{Q P}(x)=\left(\begin{array}{l}
0.643192 \\
0.514554 \\
0.450235 \\
0.8 \\
0.3
\end{array}\right)
$$

Then the error made by each method is:

$$
\begin{gathered}
d\left(\sigma_{m}, \mu\right)=0.482290 \\
d\left(\sigma_{p}, \mu\right)=0.640202 \\
d\left(\sigma_{Q P}, \mu\right)=0.434542
\end{gathered}
$$


while

$$
\begin{aligned}
& d\left(\phi_{E}(\mu), \mu\right)=0.553671 \\
& d\left(\psi_{E}(\mu), \mu\right)=0.959012
\end{aligned}
$$

Table 1 summarizes the results obtained for the Łukasievicz and the Product t-norms by all these methods.

Finally, let us illustrate an example for a non-finite set. Consider the Eukasievicz indistinguishability operator $E(x, y)=1-|x-y|$ on $[0,1]$ and let $\mu$ be the fuzzy subset of $[0,1] \mu(x)=\sqrt{x}$ which is not extensional with respect to $E$.

Then

$$
\phi_{E}(\mu)(x)=\left\{\begin{array}{lll}
x+0.25 & \text { if } 0 \leq x \leq 0.25 \\
\sqrt{x} & \text { if } \quad 0.25 \leq x \leq 1
\end{array}\right.
$$

and

$$
\psi_{E}(\mu)(x)=x .
$$

Figure 1 shows the plots of the sets $\mu(x), \phi_{E}(\mu)(x)$ and $\psi_{E}(\mu)(x)$.

Following the formulas derived in Section 6 we have that the weight for the mean-based method is $r=0.538271$. By these method we obtain the extensional fuzzy subset $\sigma_{m}$

$$
\sigma_{m}(x)=\left\{\begin{array}{lll}
x+0.134568 & \text { if } & 0 \leq x \leq 0.25 \\
0.461729 x+0.538271 \sqrt{x} & \text { if } & 0.25 \leq x \leq 1
\end{array}\right.
$$

With the power based method we find that the best solution is found for $r=0.7$, which corresponds to the extensional set $\sigma_{p}$

$$
\sigma_{p}(x)=0.7 x+0.3
$$

and the error made by these approximations is

$$
\begin{gathered}
d\left(\phi_{E}(\mu), \mu\right)=0.010417 \\
d\left(\psi_{E}(\mu), \mu\right)=0.033333 \\
d\left(\sigma_{m}, \mu\right)=0.006632 \\
d\left(\sigma_{p}, \mu\right)=0.003333 .
\end{gathered}
$$




\begin{tabular}{|c|c|c|c|c|}
\hline & \multicolumn{2}{|c|}{$T=\mathrm{E}$} & \multicolumn{2}{|c|}{$T=\Pi$} \\
\hline Method & Extensional & Error & Extensional & Error \\
\hline$\phi_{E}$ & $\left(\begin{array}{l}0.9 \\
0.7 \\
0.6 \\
0.8 \\
0.3\end{array}\right)$ & 0.538516 & $\left(\begin{array}{l}0.9 \\
0.72 \\
0.63 \\
0.8 \\
0.3\end{array}\right)$ & 0.553671 \\
\hline$\psi_{E}$ & $\left(\begin{array}{l}0.4 \\
0.4 \\
0.1 \\
0.8 \\
0.3\end{array}\right)$ & 0.509902 & $\begin{array}{l}0.142857 \\
0.142857 \\
0.1 \\
0.333333 \\
0.3\end{array}$ & 0.959012 \\
\hline$m^{r}\left(\phi_{E}, \psi_{E}\right)$ & $\begin{array}{l}0.637288 \\
0.542373 \\
0.337288 \\
0.8 \\
0.3\end{array}$ & 0.341208 & $\begin{array}{l}0.774314 \\
0.624194 \\
0.54202 \\
0.722533 \\
0.3\end{array}$ & 0.482290 \\
\hline$\psi_{E}^{r}$ & $\left(\begin{array}{l}0.504854 \\
0.504854 \\
0.257282 \\
0.834951 \\
0.422330\end{array}\right)$ & 0.475746 & $\begin{array}{l}0.463984 \\
0.463984 \\
0.403065 \\
0.64821 \\
0.621812\end{array}$ & 0.640202 \\
\hline Quadratic Prog & $\left(\begin{array}{l}0.65 \\
0.5 \\
0.35 \\
0.8 \\
0.3\end{array}\right)$ & 0.328105 & $\begin{array}{l}0.643192 \\
0.514554 \\
0.450235 \\
0.8 \\
0.3\end{array}$ & 0.434542 \\
\hline
\end{tabular}

Table 1: Table comparing the different approximations by extensional fuzzy subsets of $\mu$ proposed and their error.

\section{Concluding Remarks}

In this paper several approaches and algorithms to approximate a fuzzy subset $\mu$ by an adequate extensional one with respect to an indistinguishability operator $E$ have been proposed.

For continuous Archimedean t-norms three different methods have been 


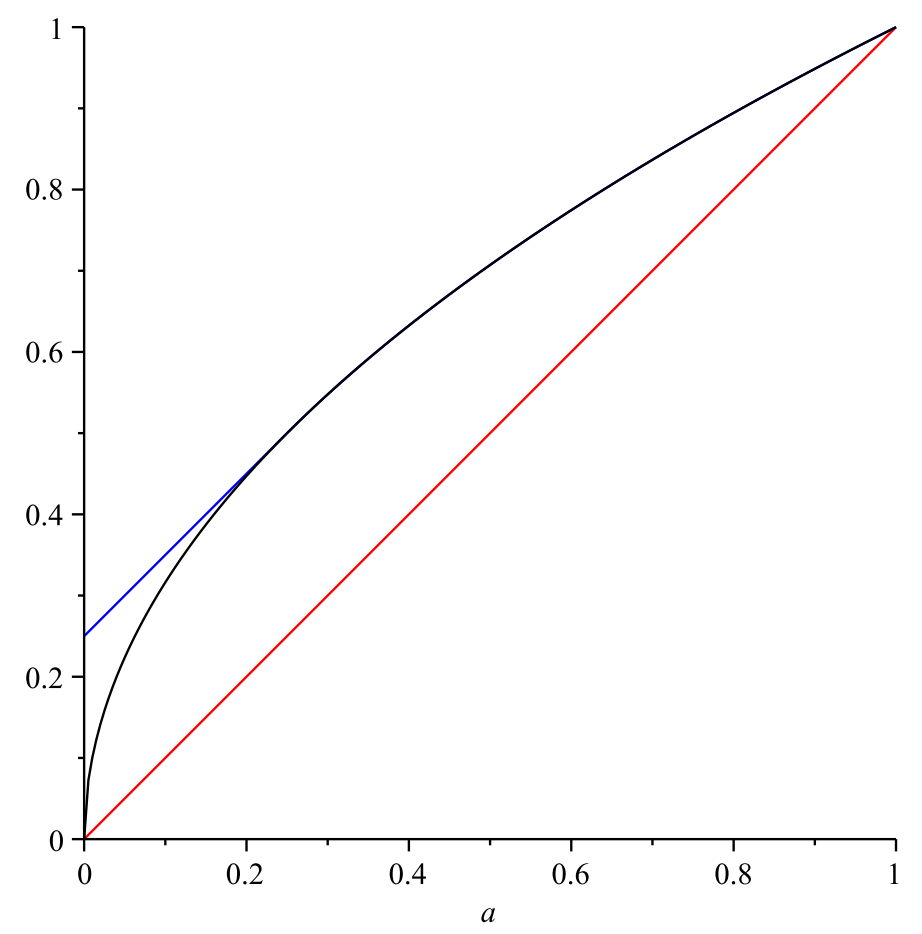

Figure 1: Plots of the sets $\mu$ (black), $\phi_{E}(\mu)$ (blue) and $\psi_{E}(\mu)$ (red).

given. The first one is based on finding an an adequate mean between the upper and the lower approximation by extensional fuzzy subsets of $\mu\left(\phi_{E}(\mu)\right.$ and $\psi_{E}(\mu)$ respectively). The second one uses powers with respect to the t-norm $T$ applied to the lower approximation operator $\psi_{E}$. Finally, the last one is based on solving a Quadratic Programming problem.

At a theoretical level the QP-based method is better than the other two as it always finds the extensional set that better approximates $\mu$, while the other two find extensional sets similar to $\mu$ but with no guarantee of being the best extensional approximation. However, the QP-based method does not work for non-finite sets and is computationally very costly, while the mean-based and the power-based ones are suitable for infinite sets and are not computationally expensive.

A practical comparison of these methods has been provided with a concrete example in Section 8. Though the results are not conclusive (a further research and study should be done to extract sure conclusions), the results of 
the example show that the quality of the power-based method is extremely dependent of the quality of the $\psi_{E}$ operator while the mean-based one is more flexible as it depends either on $\phi_{E}$ and $\psi_{E}$. In the finite set example the mean-based method finds a better approximation either with the Eukasievicz and the Product t-norm, while in the infinite example the result of the power-based one is superior.

On the other hand, when $T$ is not Archimedean the previous methods fail and other techniques have to be developed. In this paper an algorithm to find good approximations for $T=$ min has been proposed. This method is based on solving a QP problem as well. The performance of this method has been also studied in the example of Section 8 .

Finally, several lines of research could come after this work. The first one, which has already been pointed, is to compare in depth the methods proposed in order to develop a conclusive analysis of them. Alternatively, an interesting line is to find better approximations when the t-norm is not Archimedean. Finally, a different idea is to find a geometrical interpretation of the methods proposed and study their suitability to a problem with repect to the geometry of it. After all, a fuzzy sete $\mu$ is a function and thus it has a clear geometric component (if $X$ is finite set then $\mu \in[0,1]^{n}$ ). In consequence, extensional sets can be analyzed in geometric terms and either means and powers are geometric operators. An analysis of the geometry of the connection between all these concepts would be then of extreme interest and will be studied by the authors in a forthcoming work.

\section{Acknowledgment}

Research partially supported by project number TIN2009-07235.

The first author is supported by the Universitat Politècnica de Catalunya (UPC).

\section{References}

[1] Aczél, J.: Lectures on functional equations and their applications. Academic Press, NY-London (1966) 
[2] M.S. Bazaraa, H.D. Sherali, C.M. Shetty. Nonlinear programming: theory and algorithms 2nd ed. John Wiley \& Sons. New York. (1993)

[3] Bělohlávek, R.: Fuzzy Relational Systems: Foundations and Principles. Kluwer Academic Publishers, New York (2002)

[4] Boixader, D., Jacas, J., Recasens, J.: Fuzzy equivalence relations: advanced material. In: Dubois, D., Prade, H. (eds.) Fundamentals of Fuzzy Sets, pp. 261-290. Kluwer Academic Publishers, New York (2000)

[5] Boixader, D., Jacas, J., Recasens, J.: Upper and lower approximation of fuzzy sets. Int. J. of General Systems 29, 555-568 (2000)

[6] Bou, F., Esteva, F., Godo, L., Rodríguez, R.: On the Minimum ManyValued Modal Logic over a Finite Residuated Lattice. Journal of Logic and computation (2010) doi:10.1093/logcom/exp062

[7] Castro, J.L., Klawonn, F.: Similarity in Fuzzy Reasoning. Mathware \& Soft Computing 2, 197-228 (1996)

[8] J. Elorza, R. Fuentes-González, J. Bragard, P. Burillo: On The Relation Between Fuzzy Preorders, Fuzzy Closing Morphological Operators And Fuzzy Consequence Operators. Proceedings of ESTYLF2010 Conference. Huelva. 133-138 (2010)

[9] Flanders, H.: Differentiation under the integral sign. American Mathematical Monthly 80 (6): 615-627 (1973)

[10] Garmendia L., Recasens, J.: How to make T-transitive a proximity relation. IEEE Transactions on Fuzzy Systems, vol 17, issue 1, 200-207 (2009)

[11] Jacas, J., Recasens, J.: Fixed points and generators of fuzzy relations. J. Math. Anal. Appl 186, 21-29 (1994)

[12] Jacas, J., Recasens, J.: Fuzzy T-transitive relations: eigenvectors and generators. Fuzzy Sets and Systems 72, 147-154 (1995)

[13] Jacas, J., Recasens, J. Maps and Isometries between indistinguishability operators. Soft Computing 6. 14-20. (2002) 
[14] Jacas, J., Recasens, J.: Aggregation of T-Transitive Relations. Int J. of Intelligent Systems 18, 1193-1214 (2003)

[15] Klement, E.P., Mesiar, R., Pap E.: Triangular norms. Kluwer Academic Publishers, Dordrecht (2000)

[16] Mattioli, G., Recasens, J. Dualities and Isomorphisms between Indistinguishabilities and Related Concepts FUZZ'IEEE Taiwan, pp 2369 2374 . (2011).

[17] Mattioli, G., Recasens, J. Natural Means of Indistinguishability Operators. Advances in Computational Intelligence Communications in Computer and Information Science Vol 299, pp 261-270. IPMU2012 Catania (2012).

[18] Morsi, N.N., Yakout, M.M.: Axiomatics for fuzzy rough sets. Fuzzy Sets and Systems 100, 327-342 (1998)

[19] Recasens, J. Indistinguishability Operators. Modelling Fuzzy Equalities and Fuzzy Equivalence Relations Series: Studies in Fuzziness and Soft Computing, Vol. 260 (2011)

[20] Valverde, L.: On the Structure of F-indistinguishability Operators. Fuzzy Sets and Systems 17, 313-328 (1985)

[21] Williamson, J.H.: Lebesgue Integration . Holt, Rinehart and Winston (1962)

[22] Zadeh. L.A. Similarity relations and fuzzy orderings. Inform. Sci. 3, 177$200(1971)$

[23] Zadeh, L.A.: Fuzzy sets and information granularity. In: Gupta, M.M., Ragade, R.K., Yager, R.R. (eds.) Advances in Fuzzy Set Theory and Applications, pp. 3-18. Amsterdam, North-Holland (1979)

[24] Zadeh, L.A.: Toward a theory of fuzzy information granulation and its centrality in human reasoning and fuzzy logic. Fuzzy Sets and Systems 90, 111-127 (1997) 